ANNALES

POLONICI MATHEMATICI

99.1 (2010)

\title{
Uniqueness of two analytic functions sharing four values in an angular domain
}

\author{
by Hong-Yan Xu (Jingdezhen) and Ting-Bin CAO (Nanchang)
}

\begin{abstract}
We deal with the uniqueness problem for analytic functions sharing four distinct values in an angular domain and obtain some theorems which improve the result given by Cao and Yi [J. Math. Anal. Appl. 358 (2009)].
\end{abstract}

1. Introduction and main results. We use $\mathbb{C}$ to denote the open complex plane, $\widehat{\mathbb{C}}(=\mathbb{C} \cup\{\infty\})$ to denote the extended complex plane, and $X$ $(\subset \mathbb{C})$ to denote an angular domain. Let $g$ be a transcendental meromorphic (analytic) function defined in the whole $\mathbb{C}$ and not rational. It is assumed that the readers are familiar with the notations of Nevanlinna theory such as $T(r, f), m(r, f), N(r, f)$ and so on, that can be found, for instance, in [5, 14.

For $a \in \widehat{\mathbb{C}}$, if two meromorphic functions $f$ and $g$ share the value $a \mathrm{CM}$ $[\mathrm{IM}]$ in $X \subseteq \mathbb{C}$, then we say that $f(z)-a$ and $g(z)-a$ have the same zeros with the same multiplicities [ignoring multiplicities] in $X \subseteq \mathbb{C}$, and if $a$ is shared IM in $X \subseteq \mathbb{C}$ by $f$ and $g$ and the multiplicities of zeros of $f-a$ and $g-a$ are different, then we say that the value $a$ is shared DM in $X \subseteq \mathbb{C}$ by $f$ and $g$. In addition, we write $f=a \rightleftharpoons g=a$ in $X \subseteq \mathbb{C}$ to indicate that $f$ and $g$ share the value $a \mathrm{CM}$ in $X, f=a \Leftrightarrow g=a$ in $X \subseteq \mathbb{C}$ to indicate that $f$ and $g$ share the value $a$ IM in $X$, and $f=a \Rightarrow g=a$ in $X$ to indicate that $f=a$ implies $g=a$ in $X$.

R. Nevanlinna (see [10]) proved the following well-known theorem.

TheOREM 1.1 (see [10]). If $f$ and $g$ are two nonconstant meromorphic functions that share five distinct values $a_{1}, a_{2}, a_{3}, a_{4}, a_{5} I M$ in $X=\mathbb{C}$, then $f(z) \equiv g(z)$.

After his theorems, the uniqueness of meromorphic functions sharing values in the whole complex plane attracted much investigation (see [16]).

2010 Mathematics Subject Classification: 30D30, 30D35.

Key words and phrases: analytic function, angular domain, uniqueness. 
E. Mues [9] dealt with the uniqueness of analytic functions sharing values DM in the whole complex plane and proved the following theorem.

TheOREM 1.2 (see [9]). There are no two distinct nonconstant analytic functions $f$ and $g$ that share three distinct values DM in $X=\mathbb{C}$.

In [18], J. H. Zheng studied the uniqueness problem under the condition that five values are shared in some angular domain in $\mathbb{C}$. It is an interesting topic to investigate the uniqueness with shared values in the complement of an unbounded closed set in the complex plane (see 1, 2, 6, 7, 8, 12, 13, [17, 18, 19]). J. H. Zheng [14, T. B. Cao and H. X. Yi [2], and J. F. Xu and $\mathrm{H}$. X. Yi [13] continued the investigation of uniqueness of meromorphic functions sharing five values and four values, and W. C. Lin, S. Mori and K. Tohge [6] and W. C. Lin, S. Mori and H. X. Yi [7 investigated the uniqueness of meromorphic and entire functions sharing sets in an angular domain.

To state our results, we need the following basic notations and definitions for meromorphic functions in an angular domain (see [5, 18, 19]).

Let $f$ be a meromorphic function on the angular domain $\bar{\Omega}(\alpha, \beta)=\{z$ : $\alpha \leq \arg z \leq \beta\}$ with $0<\beta-\alpha \leq 2 \pi$. Define

$$
\begin{gathered}
A_{\alpha, \beta}(r, f)=\frac{\omega}{\pi} \int_{1}^{r}\left(\frac{1}{t^{\omega}}-\frac{t^{\omega}}{r^{2 \omega}}\right)\left\{\log ^{+}\left|f\left(t e^{i \alpha}\right)\right|+\log ^{+}\left|f\left(t e^{i \beta}\right)\right|\right\} \frac{d t}{t}, \\
B_{\alpha, \beta}(r, f)=\frac{2 \omega}{\pi r^{\omega}} \int_{\alpha}^{\beta} \log ^{+}\left|f\left(r e^{i \theta}\right)\right| \sin \omega(\theta-\alpha) d \theta, \\
C_{\alpha, \beta}(r, f)=2 \sum_{1<\left|b_{\mu}\right|<r}\left(\frac{1}{\left|b_{\mu}\right|^{\omega}}-\frac{\left|b_{\mu}\right|^{\omega}}{r^{2 \omega}}\right) \sin \omega\left(\theta_{\mu}-\alpha\right), \\
D_{\alpha, \beta}(r, f)=A_{\alpha, \beta}(r, f)+B_{\alpha, \beta}(r, f), \quad S_{\alpha, \beta}(r, f)=D_{\alpha, \beta}(r, f)+C_{\alpha, \beta}(r, f),
\end{gathered}
$$

where $\omega=\pi /(\beta-\alpha)$ and $b_{\mu}=\left|b_{\mu}\right| e^{i \theta_{\mu}}(\mu=1,2, \ldots)$ are the poles of $f$ on $\bar{\Omega}(\alpha, \beta)$ counted according to their multiplicities. $S_{\alpha, \beta}(r, f)$ is called the Nevanlinna angular characteristic; $C_{\alpha, \beta}(r, f)$ is the angular counting function of the poles of $f$ on $\bar{\Omega}(\alpha, \beta)$; and $\bar{C}_{\alpha, \beta}(r, f)$ is the reduced function of $C_{\alpha, \beta}(r, f)$.

In 2008, T. B. Cao and H. X. Yi [1] investigated the problem of uniqueness of transcendental analytic functions sharing three values DM in an angular domain and obtained the following result which extended Theorem 1.2 to an angular domain.

Theorem 1.3 (see [1, Theorem 1]). There are no two distinct transcendental analytic functions $f$ and $g$ that share three distinct values $a_{1}, a_{2}, a_{3}$ $D M$ in an angular domain $X=\{z: \alpha<\arg z<\beta\}$ with $0 \leq \alpha<\beta \leq 2 \pi$ 
provided that

$$
\lim _{r \rightarrow \infty} \frac{S_{\alpha, \beta}(r, f)}{\log (r T(r, f))}=\infty \quad(r \notin E) .
$$

In 2009, T. B. Cao and H. X. Yi [2] investigated the uniqueness of transcendental meromorphic functions sharing five values IM in an angular domain and obtained the following result which extended Theorem 1.1 to an angular domain.

TheOREM 1.4 (see [2, Theorem 1.3]). Let $f$ and $g$ be transcendental meromorphic functions, and $X=\{z: \alpha<\arg z<\beta\}$ with $0<\beta-\alpha \leq 2 \pi$. Assume that $f$ and $g$ share five distinct values $a_{j}(j=1,2,3,4,5)$ IM in $X$. Then $f(z) \equiv g(z)$ provided that

$$
\lim _{r \rightarrow \infty} \frac{S_{\alpha, \beta}(r, f)}{\log (r T(r, f))}=\infty \quad(r \notin E) .
$$

Moreover, T. B. Cao and H. X. Yi [2] also investigated the uniqueness of transcendental meromorphic functions $f, g$ sharing four distinct values $\mathrm{CM}$ in an angular domain $X$, and of $f, g$ sharing two distinct values $\mathrm{CM}$ in $X$ and another two distinct values IM in $X$, and they obtained two interesting results which extended the analogous results in the whole complex plane to an angular domain.

From Theorem 1.3, it is natural to ask what will happen when analytic functions $f, g$ share some values CM or IM in an angular domain.

In this paper, we investigate the above question and obtain the following results.

THEOREM 1.5. Let $f$ and $g$ be transcendental analytic functions, and $X=\{z: \alpha<\arg z<\beta\}$ with $0<\beta-\alpha \leq 2 \pi$. Assume that $f$ and $g$ share two distinct values $a_{1}, a_{2} C M$ in $X ; f=a_{3} \Rightarrow g=a_{3}$ in $X ; f=a_{4} \Rightarrow g=a_{4}$ in $X$; and

$$
\lim _{r \rightarrow \infty} \frac{S_{\alpha, \beta}(r, f)}{\log (r T(r))}=\infty \quad(r \notin E),
$$

where $T(r)=\max \{T(r, f), T(r, g)\}$. Then $f \equiv g$.

THEOREM 1.6. Let $f$ and $g$ be transcendental analytic functions, and $X=\{z: \alpha<\arg z<\beta\}$ with $0<\beta-\alpha \leq 2 \pi$. Assume that $f$ and $g$ share two distinct values $a_{1}, a_{2} I M$ in $X ; f=a_{3} \Rightarrow g=a_{3}$ in $X ; g=a_{4} \Rightarrow f=a_{4}$ in $X$; and $f, g$ satisfy (1). Then either $f \equiv g$, or

$$
f \equiv \frac{a_{3} g-a_{1} a_{2}}{g-a_{4}}, \quad a_{1}+a_{2}=a_{3}+a_{4},
$$

and $a_{3}, a_{4}$ are exceptional values of $f$ and $g$ in $X$, respectively.

REMARK 1.1. Theorems 1.5 and 1.6 improve Theorem 1.4. 
2. Some lemmas. To prove our results, we need the following lemmas.

LEMMA 2.1 (see [4, 15]). Let $f$ be a nonconstant meromorphic function on $\bar{\Omega}(\alpha, \beta)$. Then for every complex number a, we have

$$
S_{\alpha, \beta}\left(r, \frac{1}{f-a}\right)=S_{\alpha, \beta}(r, f)+\varepsilon(r, a),
$$

where $\varepsilon(r, a)=O(1)$ as $r \rightarrow \infty$.

LEMMA 2.2 (see [4, p. 138]). Let $f$ be a nonconstant meromorphic function on $\bar{\Omega}(\alpha, \beta)$. Then for any $1 \leq r<R$, we have

$$
A_{\alpha, \beta}\left(r, \frac{f^{\prime}}{f}\right) \leq K\left\{\left(\frac{R}{r}\right)^{\omega} \int_{1}^{R} \frac{\log ^{+} T(r, f)}{t^{1+\omega}} d t+\log ^{+} \frac{r}{R-r}+\log \frac{R}{r}+1\right\}
$$

and

$$
B_{\alpha, \beta}\left(r, \frac{f^{\prime}}{f}\right) \leq \frac{4 \omega}{r^{\omega}} m\left(r, \frac{f^{\prime}}{f}\right),
$$

where $\omega=\pi /(\beta-\alpha)$ and $K$ is a positive constant not depending on $r$ and $R$.

REMARK 2.1. Nevanlinna conjectured that

$$
D_{\alpha, \beta}\left(r, \frac{f^{\prime}}{f}\right)=A_{\alpha, \beta}\left(r, \frac{f^{\prime}}{f}\right)+B_{\alpha, \beta}\left(r, \frac{f^{\prime}}{f}\right)=o\left(S_{\alpha, \beta}\left(r, \frac{1}{f-a}\right)\right)
$$

when $r$ tends to $+\infty$ outside an exceptional set of finite linear measure, and he proved that $D_{\alpha, \beta}\left(r, f^{\prime} / f\right)=O(1)$ when $f$ is meromorphic in $\mathbb{C}$ and has finite order. In 1974, Gol'dberg [3] constructed a counterexample to (2). However, it follows from Lemma 2.2 that

$$
D_{\alpha, \beta}\left(r, \frac{f^{\prime}}{f}\right)=A_{\alpha, \beta}\left(r, \frac{f^{\prime}}{f}\right)+B_{\alpha, \beta}\left(r, \frac{f^{\prime}}{f}\right)=R(r, f),
$$

where $R(r, f)=O\{\log (r T(r, f))\}$ as $r \rightarrow \infty(r \notin E)$ and $E$ is a set with finite linear measure.

Throughout, we denote by $R(r, *)$ quantities satisfying

$$
R(r, *)=O(\log (r T(r, *))), \quad r \notin E,
$$

where $E$ is a set with finite linear measure.

Lemma 2.3 (see [1, Lemma 1]). Suppose that $f$ is a nonconstant meromorphic function and $X=\{z: \alpha<\arg z<\beta\}$, where $0<\beta-\alpha \leq 2 \pi$. Let $P(f)=a_{0} f^{p}+a_{1} f^{p-1}+\cdots+a_{p}\left(a_{0} \neq 0\right)$, where the coefficients $a_{j}$ $(j=0,1, \ldots, p)$ are constants, and let $b_{j}(j=1, \ldots, q, q \geq p+1)$ be distinct finite complex numbers. Then

$$
D_{\alpha, \beta}\left(r, \frac{P(f) \cdot f^{\prime}}{\left(f-b_{1}\right) \cdots\left(f-b_{q}\right)}\right)=R(r, f) .
$$


LEMmA 2.4 (see [19]). Suppose that $f$ is a nonconstant meromorphic function in $\bar{\Omega}(\alpha, \beta)$ with $0<\beta-\alpha \leq 2 \pi$. Then for arbitrary distinct $a_{j} \in \widehat{\mathbb{C}}$ $(1 \leq j \leq q)$, we have

$$
(q-2) S_{\alpha, \beta}(r, f) \leq \sum_{j=1}^{q} \bar{C}_{\alpha, \beta}\left(r, \frac{1}{f-a_{j}}\right)+R(r, f),
$$

with $\bar{C}_{\alpha, \beta}\left(r, 1 /\left(f-a_{j}\right)\right)$ replaced by $\bar{C}_{\alpha, \beta}(r, f)$ if $a_{j}=\infty$.

LEMMA 2.5. Let $f$ and $g$ be distinct transcendental analytic functions, and $X=\{z: \alpha<\arg z<\beta\}$ with $0<\beta-\alpha \leq 2 \pi$. Assume that $f$ and $g$ share $a_{1}, a_{2} I M$ in $X ; f=a_{3} \Rightarrow g=a_{3}$ in $X ; f=a_{4} \Rightarrow g=a_{4}$ in $X$; and $a_{i} \in \mathbb{C}(i=1,2,3,4)$ are four distinct finite complex numbers. Then

(i) $S_{\alpha, \beta}(r, g)=2 S_{\alpha, \beta}(r, f)+R(r)$;

(ii) $S_{\alpha, \beta}(r, f-g)=3 S_{\alpha, \beta}(r, f)+R(r)$;

(iii) $S_{\alpha, \beta}(r, f)=\bar{C}_{\alpha, \beta}\left(r, \frac{1}{f-a_{3}}\right)+\bar{C}_{\alpha, \beta}\left(r, \frac{1}{f-a_{4}}\right)+R(r)$;

(iv) $S_{\alpha, \beta}(r, f)=\bar{C}_{\alpha, \beta}\left(r, \frac{1}{f-a_{i}}\right)+R(r), i=1,2$;

(v) $S_{\alpha, \beta}(r, g)=\bar{C}_{\alpha, \beta}\left(r, \frac{1}{g-a_{i}}\right)+R(r), i=3,4$;

(vi) $S_{\alpha, \beta}\left(r, f^{\prime}\right)=S_{\alpha, \beta}(r, f)+R(r), S_{\alpha, \beta}\left(r, g^{\prime}\right)=S_{\alpha, \beta}(r, g)+R(r)$,

where $R(r)=\max \{R(r, f), R(r, g)\}$.

Proof. Since $f, g$ are distinct transcendental analytic functions and share $a_{1}, a_{2}$ IM in $X$, by Lemma 2.4 we have $S_{\alpha, \beta}(r, f) \leq S_{\alpha, \beta}(r, g)+R(r, f)$ and $S_{\alpha, \beta}(r, g) \leq S_{\alpha, \beta}(r, f)+R(r, g)$.

Let

$$
\eta:=\frac{f^{\prime} g^{\prime}(f-g)}{\left(f-a_{3}\right)\left(f-a_{4}\right)\left(g-a_{1}\right)\left(g-a_{2}\right)} .
$$

From the conditions of the lemma, we deduce that $\eta$ is analytic in $X$ and $\eta \not \equiv 0$ unless $f \equiv g$. By Lemma 2.3, we have $D_{\alpha, \beta}(r, \eta)=R(r, f)+R(r, g)=$ $R(r)$. Thus, we get $S_{\alpha, \beta}(r, \eta)=R(r)$.

Since $f, g$ are transcendental analytic functions in $X$, share $a_{1}, a_{2}$ IM in $X$, and $f=a_{3} \Rightarrow g=a_{3}$ and $f=a_{4} \Rightarrow g=a_{4}$ in $X$, again by Lemma 2.4 we have

$$
\begin{aligned}
3 S_{\alpha, \beta}(r, f) & \leq \sum_{i=1}^{4} \bar{C}_{\alpha, \beta}\left(r, \frac{1}{f-a_{i}}\right)+R(r, f) \\
& \leq \bar{C}_{\alpha, \beta}\left(r, \frac{1}{f-g}\right)+R(r, f)=S_{\alpha, \beta}(r, f-g)+R(r, f) \\
& \leq S_{\alpha, \beta}(r, f)+S_{\alpha, \beta}(r, g)+R(r)
\end{aligned}
$$


and

$$
\begin{aligned}
S_{\alpha, \beta}(r, g) & \leq \bar{C}_{\alpha, \beta}\left(r, \frac{1}{g-a_{1}}\right)+\bar{C}_{\alpha, \beta}\left(r, \frac{1}{g-a_{2}}\right)+R(r, g) \\
& =\bar{C}_{\alpha, \beta}\left(r, \frac{1}{f-a_{1}}\right)+\bar{C}_{\alpha, \beta}\left(r, \frac{1}{f-a_{2}}\right)+R(r) \\
& \leq 2 S_{\alpha, \beta}(r, f)+R(r) .
\end{aligned}
$$

From (6) and (9), we get (i); from (5), (6) and (i), we get (ii); and from (4), (6), (8), (9) and (i), we get (iii). We can easily deduce (iv) and (v) from (4)-(9) and (i)-(iii). Now, we prove (vi). First, we rewrite (3) as

$$
f=f^{\prime} \frac{g^{\prime}}{\eta\left(g-a_{1}\right)\left(g-a_{2}\right)}+\frac{f^{\prime} g^{\prime}\left(a_{3} f+a_{4} f-a_{3} a_{4}-f g\right)}{\eta\left(f-a_{3}\right)\left(f-a_{4}\right)\left(g-a_{1}\right)\left(g-a_{2}\right)} .
$$

From (10) and Lemma 2.3, we get $D_{\alpha, \beta}(r, f) \leq D_{\alpha, \beta}\left(r, f^{\prime}\right)+R(r, f)$. Since $f$ is analytic in $X$, we have $S_{\alpha, \beta}\left(r, f^{\prime}\right)=S_{\alpha, \beta}(r, f)+R(r, f)$. Similarly, $S_{\alpha, \beta}\left(r, g^{\prime}\right)=S_{\alpha, \beta}(r, g)+R(r, g)$.

3. Proof of Theorem 1.5. Suppose that $f \not \equiv g$. Then conclusions (i)-(vi) of Lemma 2.5 hold. We denote

$$
\begin{aligned}
& \psi_{1}:=\frac{f^{\prime}\left(f-a_{3}\right)}{\left(f-a_{1}\right)\left(f-a_{2}\right)}-\frac{g^{\prime}\left(g-a_{3}\right)}{\left(g-a_{1}\right)\left(g-a_{2}\right)}, \\
& \psi_{2}:=\frac{f^{\prime}\left(f-a_{4}\right)}{\left(f-a_{1}\right)\left(f-a_{2}\right)}-\frac{g^{\prime}\left(g-a_{4}\right)}{\left(g-a_{1}\right)\left(g-a_{2}\right)} .
\end{aligned}
$$

By Lemma 2.3, we get

$$
D_{\alpha, \beta}\left(r, \psi_{i}\right)=R(r, f)+R(r, g)=R(r), \quad i=1,2 .
$$

Moreover, we can prove $C_{\alpha, \beta}\left(r, \psi_{i}\right)=O(1)(i=1,2)$. In fact, the poles of $\psi_{i}$ in $X$ can only occur at the zeros of $f-a_{j}$ and $g-a_{j}(i, j=1,2)$ in $X$. Since $f, g$ share $a_{1}, a_{2} \mathrm{CM}$ in $X$, we see that if $z_{0} \in X$ is a zero of $f-a_{j}$ with multiplicity $m(\geq 1)$, then it is a zero of $g-a_{j}$ with multiplicity $m$. Suppose that

$$
f-a_{i}=\left(z-z_{0}\right)^{m} \alpha(z), \quad g-a_{i}=\left(z-z_{0}\right)^{m} \beta(z),
$$

where $\alpha(z), \beta(z)$ are analytic functions in $X$. By a simple calculation,

$$
\psi_{i}=K\left(\frac{\alpha^{\prime}(z)}{\alpha(z)}-\frac{\beta^{\prime}(z)}{\beta(z)}\right), \quad i=1,2
$$

where $K$ is a constant. Therefore, $\psi_{i}(i=1,2)$ are analytic in $X$. Thus, from (11), we get $S_{\alpha, \beta}\left(r, \psi_{i}\right)=R(r)(i=1,2)$. 
If $\psi_{i} \not \equiv 0, i=1,2$, then

$$
\begin{aligned}
& \bar{C}_{\alpha, \beta}\left(r, \frac{1}{f-a_{3}}\right) \leq C_{\alpha, \beta}\left(r, \frac{1}{\psi_{1}}\right) \leq S_{\alpha, \beta}\left(r, \psi_{1}\right)+R(r, f)=R(r), \\
& \bar{C}_{\alpha, \beta}\left(r, \frac{1}{f-a_{4}}\right) \leq C_{\alpha, \beta}\left(r, \frac{1}{\psi_{2}}\right) \leq S_{\alpha, \beta}\left(r, \psi_{2}\right)+R(r, f)=R(r) .
\end{aligned}
$$

From (12), (13) and Lemma 2.5(iv), we have $S_{\alpha, \beta}(r, f) \leq R(r)$. Thus, from (1) and the definition of $R(r)$, we get a contradiction.

Assume that one of $\psi_{1}$ and $\psi_{2}$ is identically zero, say $\psi_{1} \equiv 0$. Then

$$
\bar{C}_{\alpha, \beta}^{(2}\left(r, \frac{1}{g-a_{4}}\right)=\bar{C}_{\alpha, \beta}^{(2}\left(r, \frac{1}{f-a_{4}}\right),
$$

where $\bar{C}_{\alpha, \beta}^{(2}\left(r, \frac{1}{f-a_{4}}\right)$ is the counting function of the distinct zeros of $f-a_{4}$ in $X$ with multiplicity $q \geq 2$, and similarly for $\bar{C}_{\alpha, \beta}^{(2}\left(r, \frac{1}{g-a_{4}}\right)$.

From (3), we see that $g\left(z_{1}\right)=a_{4}$ implies $f\left(z_{1}\right)=a_{4}$ for $z_{1} \in X$ satisfying $\eta\left(z_{1}\right) \neq 0$. Since $S_{\alpha, \beta}(r, \eta)=R(r)$, we have

$$
\bar{C}_{\alpha, \beta}^{1)}\left(r, \frac{1}{g-a_{4}}\right)=\bar{C}_{\alpha, \beta}^{1)}\left(r, \frac{1}{f-a_{4}}\right)+R(r),
$$

where $\bar{C}_{\alpha, \beta}^{1)}\left(r, \frac{1}{f-a_{4}}\right)$ is the counting function of the distinct simple zeros of $f-a_{4}$ in $X$, and similarly for $\bar{C}_{\alpha, \beta}^{1)}\left(r, \frac{1}{g-a_{4}}\right)$.

From (14) and (15), we get

$$
\bar{C}_{\alpha, \beta}\left(r, \frac{1}{g-a_{4}}\right)=\bar{C}_{\alpha, \beta}\left(r, \frac{1}{f-a_{4}}\right)+R(r) .
$$

Similarly, when $\psi_{2} \equiv 0$, we get

$$
\bar{C}_{\alpha, \beta}\left(r, \frac{1}{g-a_{3}}\right)=\bar{C}_{\alpha, \beta}\left(r, \frac{1}{f-a_{3}}\right)+R(r) .
$$

From (16) or (17) and Lemma 2.5(i)\&(v), we get

$$
2 S_{\alpha, \beta}(r, f)=\bar{C}_{\alpha, \beta}\left(r, \frac{1}{f-a_{3}}\right)+R(r)
$$

or

$$
2 S_{\alpha, \beta}(r, f)=\bar{C}_{\alpha, \beta}\left(r, \frac{1}{f-a_{4}}\right)+R(r) .
$$

Therefore, from (1) and the definition of $R(r)$, we get a contradiction again. Thus, we complete the proof of Theorem 1.5. 
4. Proof of Theorem 1.6. Suppose that $f \not \equiv g$. By Lemma 2.4, we have

$$
\begin{aligned}
2 S_{\alpha, \beta}(r, f)+ & \bar{C}_{\alpha, \beta}\left(r, \frac{1}{g-a_{4}}\right) \\
\leq & \bar{C}_{\alpha, \beta}\left(r, \frac{1}{f-a_{1}}\right)+\bar{C}_{\alpha, \beta}\left(r, \frac{1}{f-a_{2}}\right)+\bar{C}_{\alpha, \beta}\left(r, \frac{1}{f-a_{3}}\right) \\
& +\bar{C}_{\alpha, \beta}\left(r, \frac{1}{g-a_{4}}\right)+R(r, f) \\
\leq & \bar{C}_{\alpha, \beta}\left(r, \frac{1}{f-g}\right)+R(r, f) \leq S_{\alpha, \beta}(r, f)+S_{\alpha, \beta}(r, g)+R(r) .
\end{aligned}
$$

Therefore,

$$
S_{\alpha, \beta}(r, f)+\bar{C}_{\alpha, \beta}\left(r, \frac{1}{g-a_{4}}\right) \leq S_{\alpha, \beta}(r, g)+R(r) .
$$

Similarly,

$$
S_{\alpha, \beta}(r, g)+\bar{C}_{\alpha, \beta}\left(r, \frac{1}{f-a_{3}}\right) \leq S_{\alpha, \beta}(r, f)+R(r) .
$$

From (18) and (19), we see that $S_{\alpha, \beta}(r, f)=S_{\alpha, \beta}(r, g)+R(r)$, and

$$
\bar{C}_{\alpha, \beta}\left(r, \frac{1}{f-a_{3}}\right)=R(r), \quad \bar{C}_{\alpha, \beta}\left(r, \frac{1}{g-a_{4}}\right)=R(r) .
$$

Thus, we get

$$
2 S_{\alpha, \beta}(r, f)=\bar{C}_{\alpha, \beta}\left(r, \frac{1}{f-a_{1}}\right)+\bar{C}_{\alpha, \beta}\left(r, \frac{1}{f-a_{2}}\right)+R(r) .
$$

From (21), we can see that "almost all" zeros of $f-a_{i}(i=1,2)$ in $X$ are simple. Similarly, "almost all" zeros of $g-a_{i}(i=1,2)$ in $X$ are simple. Let

$$
\begin{aligned}
\varphi_{1} & :=\frac{\left(a_{1}-a_{3}\right) f^{\prime}\left(f-a_{2}\right)}{\left(f-a_{1}\right)\left(f-a_{3}\right)}-\frac{\left(a_{1}-a_{4}\right) g^{\prime}\left(g-a_{2}\right)}{\left(g-a_{1}\right)\left(g-a_{4}\right)}, \\
\varphi_{2} & :=\frac{\left(a_{2}-a_{3}\right) f^{\prime}\left(f-a_{1}\right)}{\left(f-a_{2}\right)\left(f-a_{3}\right)}-\frac{\left(a_{2}-a_{4}\right) g^{\prime}\left(g-a_{1}\right)}{\left(g-a_{2}\right)\left(g-a_{4}\right)} .
\end{aligned}
$$

By Lemma 2.3, we get $D_{\alpha, \beta}\left(r, \varphi_{i}\right)=R(r)(i=1,2)$. Since $f, g$ share $a_{1}, a_{2}$ IM in $X$ and from (20), we have $C_{\alpha, \beta}\left(r, \varphi_{i}\right)=R(r)(i=1,2)$. Therefore, $S_{\alpha, \beta}\left(r, \varphi_{i}\right)=R(r)(i=1,2)$.

If $\varphi_{1} \equiv 0$, then $\bar{C}_{\alpha, \beta}\left(r, \frac{1}{f-a_{2}}\right) \leq \bar{C}_{\alpha, \beta}\left(r, \frac{1}{\varphi_{1}}\right)=R(r)$. Thus, from (21), we easily get a contradiction. Similarly, when $\varphi_{2} \equiv 0$, we get a contradiction, too. Hence, $\varphi_{1}, \varphi_{2}$ are identically zero. Then we have $\frac{\varphi_{1}-\varphi_{2}}{a_{1}-a_{2}} \equiv 0$, i.e.,

$$
\frac{f^{\prime}}{f-a_{3}}-\frac{g^{\prime}}{g-a_{4}}-\frac{f^{\prime}}{f-a_{1}}+\frac{g^{\prime}}{g-a_{1}}-\frac{f^{\prime}}{f-a_{2}}+\frac{g^{\prime}}{g-a_{2}} \equiv 0,
$$


which implies that

$$
\frac{f-a_{3}}{g-a_{4}} \cdot \frac{\left(g-a_{1}\right)\left(g-a_{2}\right)}{\left(f-a_{1}\right)\left(f-a_{2}\right)} \equiv c,
$$

where $c$ is a nonzero constant. Rewrite (22) as

$$
g^{2}-\left(a_{1}+a_{2}-\frac{c \gamma(f)}{f-a_{3}}\right) g+a_{1} a_{2}+\frac{c a_{4} \gamma(f)}{f-a_{3}} \equiv 0,
$$

where $\gamma(f):=\left(f-a_{1}\right)\left(f-a_{2}\right)$. The discriminant of $(23)$ is

$$
\Delta(f)=\left(a_{1}+a_{2}-\frac{c \gamma(f)}{f-a_{3}}\right)^{2}-4\left(a_{1} a_{2}+\frac{c a_{4} \gamma(f)}{f-a_{3}}\right)=\frac{Q(f)}{\left(f-a_{3}\right)^{2}},
$$

where

$$
Q(z):=\left(\left(a_{1}+a_{2}\right)\left(z-a_{3}\right)-c \gamma(z)\right)^{2}-4 a_{1} a_{2}\left(z-a_{3}\right)^{2}-4 c a_{4} \gamma(z)\left(z-a_{3}\right)
$$

is a polynomial of degree 4 in $z$. If $a$ is a zero of $Q(z)$ in $X$, obviously $a \neq a_{3}$. Then from (23), $f(z)=a$ implies that

$$
g(z)=\frac{1}{2}\left(a_{1}+a_{2}-\frac{c \gamma(a)}{a-a_{3}}\right)=: b .
$$

Set

$$
\begin{aligned}
\phi_{1} & :=\frac{f^{\prime} g^{\prime}(f-g)}{\left(f-a_{1}\right)\left(g-a_{2}\right)\left(f-a_{3}\right)\left(g-a_{4}\right)}, \\
\phi_{2} & :=\frac{f^{\prime} g^{\prime}(f-g)}{\left(f-a_{2}\right)\left(g-a_{1}\right)\left(f-a_{3}\right)\left(g-a_{4}\right)}, \\
\phi & :=\frac{\phi_{2}}{\phi_{1}}=\frac{\left(f-a_{1}\right)\left(g-a_{2}\right)}{\left(f-a_{2}\right)\left(g-a_{1}\right)} .
\end{aligned}
$$

By Lemma 2.3, we get $D_{\alpha, \beta}\left(r, \phi_{i}\right)=R(r)(i=1,2)$, and by a simple calculation, $C_{\alpha, \beta}\left(r, \phi_{i}\right)=R(r)(i=1,2)$. Then $S_{\alpha, \beta}\left(r, \phi_{i}\right)=R(r)(i=1,2)$, and thus $S_{\alpha, \beta}(r, \phi)=R(r)$.

Assume that $f$ is not a Möbius transformation of $g$. Then $\phi$ is a nonconstant function. Since

$$
\begin{aligned}
& Q\left(a_{1}\right)=\left(a_{1}-a_{3}\right)^{2}\left(a_{1}-a_{2}\right)^{2} \neq 0, \\
& Q\left(a_{2}\right)=\left(a_{2}-a_{3}\right)^{2}\left(a_{1}-a_{2}\right)^{2} \neq 0,
\end{aligned}
$$

from $a \neq a_{i}(i=1,2)$ and (22), we get

$$
\bar{C}_{\alpha, \beta}\left(r, \frac{1}{f-a}\right) \leq \bar{C}_{\alpha, \beta}\left(r, \frac{1}{\phi-\xi}\right) \leq S_{\alpha, \beta}(r, \phi)=R(r)
$$

where $\xi=\frac{\left(a-a_{1}\right)\left(b-a_{2}\right)}{\left(a-a_{2}\right)\left(b-a_{1}\right)}$. Since $f$ is analytic in $X$, and by Lemma 2.4 and (20), we get

$$
S_{\alpha, \beta}(r, f) \leq \bar{C}_{\alpha, \beta}\left(r, \frac{1}{f-a_{3}}\right)+\bar{C}_{\alpha, \beta}\left(r, \frac{1}{f-a}\right)+R(r)=R(r)
$$


The above inequality contradicts (1). Therefore $f$ is a Möbius transformation of $g$. Since $f, g$ are analytic functions in $X$, by a simple calculation we can easily get $a_{1}+a_{2}=a_{3}+a_{4}$ and

$$
f \equiv \frac{a_{3} g-a_{1} a_{2}}{g-a_{4}}
$$

furthermore, $a_{3}, a_{4}$ are exceptional values of $f$ and $g$ in $X$, respectively.

Thus, we complete the proof of Theorem 1.6.

Acknowledgments. This research was supported by the Youth Foundation of Education Department of Jiangxi (grant no. GJJ10223 and no. GJJ10050), China.

\section{References}

[1] T. B. Cao and H. X. Yi, Analytic functions sharing three values DM in one angular domain, J. Korean Math. Soc. 45 (2008), 1523-1534.

[2] - - - On the uniqueness of meromorphic functions that share four values in one angular domain, J. Math. Anal. Appl. 358 (2009), 81-97.

[3] A. A. Gol'dberg, Nevanlinna's lemma on the logarithmic derivative of a meromorphic function, Math. Notes 17 (1975), 310-312.

[4] A. A. Gol'dberg and I. V. Ostrovskiǔ, The Distribution of Values of Meromorphic Functions, Nauka, Moscow, 1970 (in Russian).

[5] W. K. Hayman, Meromorphic Functions, Oxford Univ. Press, London, 1964.

[6] W. C. Lin, S. Mori and K. Tohge, Uniqueness theorems in an angular domain, Tohoku Math. J. 58 (2006), 509-527.

[7] W. C. Lin, S. Mori and H. X. Yi, Uniqueness theorems of entire functions with shared-set in an angular domain, Acta Math. Sinica 24 (2008), 1925-1934.

[8] Z. Q. Miao and H. F. Liu, Meromorphic functions in the unit disc that share values in an angular domain, J. Math. Anal. Appl., doi:10.1016/j.jmaa.2009.05.043.

[9] E. Mues, Bemerkungen zum vier-Punkte-Satz, in: Complex Methods on Partial Differential Equations, Math. Res. 53, Akademie-Verlag, Berlin, 1989, 109-117.

[10] R. Nevanlinna, Le théorème de Picard-Borel et la théorie des fonctions méromorphes, reprint of the 1929 original, Chelsea, New York, 1974.

[11] M. Reinders, A new characterization of Gundersen's example of two meromorphic functions sharing four values, Results Math. 24 (1993), 174-179.

[12] Z. J. Wu and D. C. Sun, A remark on uniqueness theorems in an angular domain, Proc. Japan Acad. Ser. A 64 (2008), no. 6, 73-77.

[13] J. F. Xu and H. X. Yi, On uniqueness of meromorphic functions with shared four values in some angular domains, Bull. Malays. Math. Sci. Soc. 31 (2008), 57-65.

[14] L. Yang, Value Distribution Theory, Springer, Berlin, and Science Press, Beijing, 1993.

[15] L. Yang and C. C. Yang, Angular distribution of $f f^{\prime}$, Sci. China Ser. A 37 (1994), $284-294$.

[16] H. X. Yi and C. C. Yang, Uniqueness Theory of Meromorphic Functions, Science Press/Kluwer, Beijing, 2003.

[17] Q. C. Zhang, Meromorphic functions sharing values in an angular domain, J. Math. Anal. Appl. 349 (2009), 100-112. 
[18] J. H. Zheng, On uniqueness of meromorphic functions with shared values in some angular domains, Canad J. Math. 47 (2004), 152-160.

[19] —, On uniqueness of meromorphic functions with shared values in one angular domains, Complex Var. Elliptic Equations 48 (2003), 777-785.

Hong-Yan $\mathrm{Xu}$ (corresponding author)

Department of Informatics and Engineering

Jingdezhen Ceramic Institute (XiangHu XiaoQu)

Jingdezhen, Jiangxi 333403, China

E-mail: xhyhhh@126.com
Ting-Bin Cao Department of Mathematics Nanchang University Nanchang, Jiangxi 330031, China E-mail: tbcao@ncu.edu.cn or ctb97@163.com

Received 9.9.2009

and in final form 19.11.2009 
